\title{
Manipulating Solid-Liquid Interfacial Interactions for Designing Surfaces with Special Wettability for Separation Processes
}

\author{
Gibum Kwon, PhD. \\ Department of Mechanical Engineering, University of Kansas, Lawrence, \\ Kansas 66045 USA \\ gbkwon@ku.edu
}

\begin{abstract}
A large volume of research efforts has thus far been devoted to developing functional surfaces by tailoring the chemistry and morphology at the molecular, nano-, and micro-scales to achieve surfaces and interfaces with desired properties. In particular, experimental and theoretical findings have demonstrated that tailoring the solid-liquid interfacial interactions such as wettability and sorption plays a pivotal role in diversifying surfaces across versatile real-world applications. In our past research, we have focused on establishing a fundamental understanding of the interactions at the interfaces between solids, liquids, and gases. These efforts have resulted in new surfaces that enable the separation of the liquid mixture, both miscible and immiscible. In this talk, first the principles of designing surfaces with customized liquid wettability, and their applications in membrane-based separations will be discussed. Subsequently, our recent achievements on responsive hydrogels and zwitterionic polymers and their potential applications in energy, national security, petrochemical, and environmental application will be discussed.
\end{abstract}

